\title{
Blood count-derived inflammatory markers predict time to Bacillus Calmette-Guérin failure in high-risk non-muscle-invasive bladder cancer
}

\author{
Aleksander Ślusarczyk, Piotr Zapała, Karolina Garbas, Łukasz Zapała, Tomasz Borkowski, \\ Piotr Radziszewski
}

Department of General, Oncological and Functional Urology, Medical University of Warsaw, Poland

Submitted: 1 September 2020

Accepted: 8 November 2020

Arch Med Sci

DOI: https://doi.org/10.5114/aoms/130303

Copyright $\odot 2021$ Termedia \& Banach

\section{Abstract}

Introduction: Bacillus Calmette-Guérin (BCG) treatment failure remains a significant clinical problem in patients with high-risk non-muscle-invasive bladder cancer (NMIBC). The European Organization for Research and Treatment of Cancer (EORTC) and Spanish Urological Club for Oncological Treatment (CUETO) risk scores remain the most commonly used models in the prediction of NMIBC recurrence and progression. On the other hand, well-established predictors of BCG failure are still lacking. Our aim was to evaluate the utility of blood count-derived inflammatory markers for BCG failure prediction in patients with high-risk NMIBC.

Material and methods: One hundred and eighty-three consecutive patients with high-risk NMIBC, who underwent transurethral resection of the bladder tumour (TURBT) and were further treated with BCG instillations were included in the study. Bacillus Calmette-Guérin failure was retrospectively determined based on European Association of Urology 2019 guidelines. Differences in BCG failure-free survival were assessed using the log-rank test. Logistic regression was utilized for uni- and multivariate analysis.

Results: Kaplan-Meier analysis revealed that patients with a high preoperative neutrophil-to-lymphocyte ratio (NLR > 2.3), platelet-to-lymphocyte ratio (PLR > 147), neutrophil-to-erythrocyte ratio (NER > 0.93), higher systemic inflammatory marker (SIM) score and with a low lymphocyte-to-monocyte ratio $(L M R<2.55)$ had shorter time to $B C G$ failure $(p<0.05)$. In the multivariate model, all markers except for LMR remained a significant adjunct to the CUETO recurrence risk score when predicting BCG failure.

Conclusions: Our study demonstrates the utility of blood count-derived inflammatory markers in the prediction of BCG failure in high-risk NMIBC. Implementation of NLR, PLR, NER and SIM score might be of clinical value especially when combined with the CUETO scoring system.

Key words: neutrophil-to-lymphocyte ratio, non-muscle-invasive bladder cancer, BCG failure, BCG unresponsive, systemic inflammatory markers.

\section{Introduction}

Bladder cancer is the $11^{\text {th }}$ most commonly diagnosed cancer worldwide [1]. Approximately $75 \%$ of patients present with a malignancy confined to the mucosa (stage Ta, CIS) or submucosa (T1) [1]. Non-muscleinvasive bladder cancer (NMIBC) represents a heterogeneous group

\author{
Corresponding author: \\ Piotr Zapala \\ Department of General, \\ Oncological and Functional \\ Urology \\ Medical University of Warsaw \\ 4 Linleya St. \\ 02-005 Warsaw, Poland \\ E-mail: \\ zapala.piotrek@gmail.com
}


of tumours with different risks of recurrence and progression [2, 3]. T1 tumours, high-grade $(\mathrm{HG})$ tumours, carcinoma in situ (CIS) and multiple, recurrent and large $(\geq 3 \mathrm{~cm})$ low-grade $(\mathrm{LG})$ tumours are considered to be at high risk of progression to muscle-invasive bladder cancer (MIBC) [1]. Conservative treatment of high-risk NMIBC includes complete transurethral resection of the bladder tumour (TURBT) followed by intravesical Bacillus Calmette-Guérin (BCG) instillations for 1 to 3 years, which constitutes a safe therapeutic standard [4, 5]. However, BCG therapy has been found to be unsuccessful within a certain group of patients due to either severe side effects (approximately $20 \%$ experience BCG intolerance) or no response to treatment (BCG failure) [6-9]. Bacillus Calmette-Guérin instillations are associated with local (cystitis, haematuria, frequency, granulomatous prostatitis, epididymo-orchitis, ureteral obstruction, contracted bladder) or systemic side effects (fever, general malaise, sepsis, allergy, arthritis) related to infection or hypersensitivity $[10,11]$. The majority of side effects occur at the beginning of the treatment (induction and early maintenance) and further maintenance schedule continuation does not increase the risk of BCG toxicity $[9,10]$. Serious side effects occur in $<5 \%$ of patients and can be managed effectively in almost all cases [9]. The most common side effects include chemical or bacterial cystitis, which can be treated with analgesics, spasmolytics or alternative therapies (e.g. hyaluronic acid instillations) [10, 12]. Unfortunately, BCG failure is observed in up to $40 \%$ of cases $[7,13]$. The recommended option for the treatment of these individuals is immediate radical cystectomy (RC). Ongoing trials evaluating the efficacy of immune checkpoint inhibitors in combination with BCG might become an alternative bladder-preserving strategy for patients who failed intravesical BCG [14]. Nonetheless, patients who experience progression to MIBC have worse cancer-specific survival compared with those primarily diagnosed with an invasive malignancy $[15,16]$. Predicting disease recurrence and progression is, thus, vital in order to separate the patients most likely to benefit from the BCG therapy from those in whom immediate RC should be indicated. To date several predictive tools to facilitate the risk stratification have been proposed based on standard clinicopathological features [2, 17]. Although Spanish Urological Club for Oncological Treatment (CUETO) scoring model and European Organization for Research and Treatment of Cancer (EORTC) risk tables are now part of routine clinical practice, their implementation in predicting BCG failure is questionable, whereas the accuracy of the novel tools remains insufficient [3]. Recent studies sug- gest that neutrophil-to-lymphocyte ratio (NLR), systemic inflammatory markers and preoperative anaemia may serve as promising predictors of recurrence and progression in patients with NMIBC [18-20]. The aim of our study was to re-evaluate the blood count-derived inflammatory markers and standard clinicopathological features as BCG failure risk factors.

\section{Material and methods}

\section{Study design and inclusion criteria}

This is a retrospective, single tertiary centre study. Medical records of 201 consecutive patients with high-risk NMIBC, who underwent TURBT between 2011 and 2018 and were further treated with BCG instillations, were reviewed. Patients with BCG for intermediate-risk NMIBC $(n=9)$, upfront metastases $(n=1)$ as well as patients who had not completed the full induction course due to intolerance $(n=8)$ were excluded.

\section{Treatment}

The procedures were carried out according to the protocol recommended by the European Association of Urology (EAU) clinical guidelines [1]. Surgical specimens were reviewed by a genitourinary pathologist, graded according to the 1973 and 2004 WHO grading system and staged according to the 2009 TNM classification.

Patients with high-risk NMIBC defined as highgrade tumour or T1 stage or CIS or multiple, recurrent and large $(\geq 3 \mathrm{~cm})$ low-grade Ta tumours were qualified for BCG in a standard schedule. All patients received an induction course of 6 weekly instillations. The maintenance schedule included 3 instillations of intravesical BCG each week at 3 , $6,12,18,24,30$ and 36 months.

\section{Follow-up and endpoint}

Follow-up included cystoscopy and urine cytology performed on a regular basis (every 3 months for the first 2 years and every 6 months from the $2^{\text {nd }}$ to the $5^{\text {th }}$ year). Suspicion of recurrence or progression raised based on follow-up was each time verified with TURBT or transurethral bladder biopsy.

The endpoint of the study was defined as histopathologically confirmed BCG failure. Bacillus Calmette-Guérin failure was defined as progression to MIBC or presence of BCG unresponsive disease (BCG refractory or early relapse within 6 months of last BCG exposure) according to the EAU 2019 guidelines [1]. Therefore, patients for whom further BCG re-induction remained a treatment option were not classified as failures. The endpoint defined as described was evaluated retrospectively. 
Eight patients were not classified as BCG failures despite high-grade disease presence after the induction course. Out of these, 3 patients experienced CIS and 5 patients TaHG persistence after the induction course. With further BCG maintenance therapy, all of them achieved disease eradication. Consequently, in accordance with current EAU guidelines the above individuals did not meet the criteria for BCG failure [1].

\section{Descriptive variables and statistical analysis}

Descriptive variables included clinical, histopathological and laboratory data. Histopathological data included primary staging and grading. Clinical data included tumour size and multifocality, age, gender, previous history of bladder cancer and comorbidities. Laboratory data included pretreatment blood parameters - blood cell counts and haemoglobin level collected from preoperative laboratory evaluation. Derived ratios between different parameters were calculated. The optimal cut-off value for each parameter was determined by the receiver operating characteristic (ROC) curve with J statistic. The systemic inflammatory marker (SIM) score was calculated based on categorized values of NLR, platelet-to-lymphocyte ratio $(P L R)$, and lymphocyte-to-monocyte ratio (LMR), as reported previously [19].

Continuous variables are presented as median values accompanied by values of the lower and upper quartile. For prediction analysis univariable and multivariable logistic regression was implemented. The differences in time to BCG failure were evaluated with the log-rank test and illustrated with Kaplan-Meier curves in the entire cohort and in predefined subgroups. To aid choice of variables for multivariate model development Spearman correlation was implemented. Differences between groups were evaluated with the Mann-Whitney $U$ test for continuous variables and with Fisher's exact test for categorical variables. For all statistical analyses, a two-sided $p$-value $<0.05$ was considered statistically significant. Statistical analyses were performed with the SAS System (version 9.4).

\section{Results}

Among 183 patients with high-risk NMIBC included in the study, 39 individuals $(21.3 \%)$ developed BCG failure. In total, 15 patients (8.2\%) progressed to muscle-invasive disease and 40 patients (21.9\%) developed high-grade tumour recurrence, including 8 (4.4\%) with high-grade persistent disease after the induction course. In 8 other individuals (4.4\%) high-grade recurrence was followed by progression to MIBC. Baseline characteristics of the cohort are presented in Table I.
Overall, 72 patients (39.3\%) completed the 1-year BCG maintenance schedule. Median cancerfree follow-up in BCG responders was 11.3 months (range between quartiles 6-19.9 months). Median time to treatment failure in BCG nonresponders was 6.5 months (range between quartiles 3-10.5 months).

\section{Uni- and multivariate analysis}

Kaplan-Meier analysis for the whole cohort revealed that patients with preoperative NLR $>2.3$ $(p<0.05)$, PLR > $147(p<0.01)$, LMR $<2.55$ $(p<0.05)$ and higher SIM score $(p<0.01)$ had significantly shorter time to BCG failure (Figure $1 \mathrm{~A}$ ).

As univariate analysis and Kaplan-Meier curves revealed an association between low red blood cell count $\left(<4.2 \times 10^{12} / l\right)$ and BCG failure $(p<0.01)$ (Fig. $1 \mathrm{~A}$ ), we derived the neutrophil-to-erythrocyte (NER) ratio as a new marker of systemic inflammation. Patients with higher preoperative NER ( $>0.93$ ) had shorter time to BCG failure compared to individuals with lower NER $(p<0.05)$ (Fig. 1 A).

In univariate logistic regression analysis systemic inflammatory markers as well as CUETO recurrence and progression risk scores, tumour multiplicity, and presence of residual tumour on reTURBT were associated with BCG failure (Table II A). Multivariate models based on the CUETO recurrence risk score are presented in Table II B. The CUETO progression risk score was not utilized to develop a multivariate model as it showed a significant correlation with NLR $(r=0.17, p<0.05)$ and SIM score $(r=0.22, p<0.05)$.

\section{Subgroups with NLR significance}

For subgroup analysis validation we chose NLR as the most conservative and extensively analysed previously (Fig. 1 B). Patients with NLR > 2.3 had shorter time to BCG failure in the subgroup of high CUETO progression risk score (10-14) $(p<0.01)$. Moreover, NLR > 2.3 remained significantly associated with time to BCG failure in patients with papillary tumour ( $\mathrm{T} 1$ and $\mathrm{Ta})(p<0.05)$ and in the subgroups of patients with recurrent tumour $(p<0.001)$, single tumour $(p<0.05)$ and tumour size $<3 \mathrm{~cm}(p<0.001)$.

Since red blood cell count revealed a significant association with BCG failure (Fig. 1 A), we also investigated whether stratification based on RBC influences the prognostic value of NLR. Kaplan-Meier analysis demonstrated that patients with NLR > 2.3 had shorter time to BCG failure only in the subgroup with normal $\operatorname{RBC}\left(\geq 4.2 \times 10^{12} / \mathrm{l}\right)$ $(p<0.01)$.

Inflammatory markers (including NLR, PLR, LMR, SIM score) remained significant predictors of BCG failure in the subgroup of patients without com- 
Table I. Clinicopathologic features of the entire cohort (183 patients)

\begin{tabular}{|c|c|c|}
\hline Factor & $n(\%)$ & Median (range) \\
\hline \multicolumn{3}{|l|}{ Gender } \\
\hline Male & $140(77)$ & - \\
\hline Female & $43(23)$ & - \\
\hline Age & - & $72(64-79)$ \\
\hline \multicolumn{3}{|l|}{ Tumour category } \\
\hline T1 & $121(66)$ & - \\
\hline $\mathrm{Ta}$ & $34(19)$ & - \\
\hline CIS & $28(15)$ & - \\
\hline \multicolumn{3}{|l|}{ Grade } \\
\hline Low & $11(6)$ & - \\
\hline High & $172(94)$ & - \\
\hline \multicolumn{3}{|l|}{ Concomitant CIS } \\
\hline No & $148(81)$ & - \\
\hline Yes & $35(19)$ & - \\
\hline \multicolumn{3}{|l|}{ Recurrence rate } \\
\hline Primary & $127(69)$ & - \\
\hline Recurrent & $56(31)$ & - \\
\hline \multicolumn{3}{|l|}{ Multiplicity } \\
\hline Solitary & $126(69)$ & - \\
\hline Multiple & $57(31)$ & - \\
\hline \multicolumn{3}{|l|}{ Size } \\
\hline$<3 \mathrm{~cm}$ & $134(73)$ & - \\
\hline$\geq 3 \mathrm{~cm}$ & $49(27)$ & - \\
\hline \multicolumn{3}{|l|}{ re-TURBT status } \\
\hline No re-TURBT & $52(28)$ & - \\
\hline No tumour & $66(36)$ & - \\
\hline Residual tumour & $65(36)$ & - \\
\hline \multicolumn{3}{|l|}{ Muscle in TURBT } \\
\hline Yes & $148(81)$ & - \\
\hline No & $35(19)$ & - \\
\hline Diabetes mellitus & $42(23)$ & - \\
\hline $\begin{array}{l}\text { CUETO recurrence risk } \\
\text { score }\end{array}$ & - & $7(5-9)$ \\
\hline $\begin{array}{l}\text { CUETO progression risk } \\
\text { score }\end{array}$ & - & $9(8-10)$ \\
\hline Neutrophil count $\left[10^{3} / \mathrm{ml}\right]$ & - & $4.57(3.6-5.87)$ \\
\hline Lymphocyte count $\left[10^{3} / \mathrm{ml}\right]$ & - & $1.81(1.40-2.36)$ \\
\hline Platelet count $\left[10^{3} / \mathrm{ml}\right]$ & - & $218(177-261)$ \\
\hline
\end{tabular}

Table I. Cont.

\begin{tabular}{|lcc|}
\hline Factor & $n$ (\%) & Median (range) \\
\hline Monocyte count $\left[10^{3} / \mathrm{ml}\right]$ & - & $0.66(0.54-0.79)$ \\
\hline RBC $\left[10^{6} / \mathrm{ml}\right]$ & - & $4.49(4.09-4.88)$ \\
\hline Haemoglobin $[\mathrm{g} / \mathrm{dl}]$ & - & $13.7(12.3-14.6)$ \\
\hline NLR & - & $2.56(1.82-3.44)$ \\
\hline PLR & - & $118(91-160)$ \\
\hline LMR & - & $2.73(2.06-3.80)$ \\
\hline NER & - & $1.01(0.80-1.34)$ \\
\hline SIM score & $65(36)$ & \\
\hline 0 & $39(21)$ & - \\
\hline 1 & $41(22)$ & - \\
\hline 2 & $38(21)$ & - \\
\hline 3 & & \\
\hline
\end{tabular}

NLR - neutrophil count/lymphocyte count, NER - neutrophil count/ red blood cell count, PLR - platelet count/lymphocyte count, $L M R$ lymphocyte count/monocyte count, SIM score $=N L R+P L R+L M R$

mon systemic diseases such as diabetes mellitus or metabolic syndrome (Supplementary Table I and Supplementary Figure 1).

To determine potential confounders, patients' baseline characteristics according to NLR status were evaluated (for details see Supplementary Table II). There were no significant differences in terms of tumour stage, grade, recurrence rate, multiplicity, tumour size, presence of concomitant $\mathrm{CIS}$, gender or median age between groups with high NLR (> 2.3) and low NLR ( $\leq 2.3)$.

\section{Discussion}

Development of dedicated BCG failure predictive models remains an unmet clinical necessity $[14,21]$. In fact, BCG failure prediction might become even more significant in the light of awaited results of clinical trials evaluating the efficacy of BCG and immune checkpoint inhibitors in highrisk NMIBC [14]. Defining individuals at higher risk of BCG failure provides a possibility to avoid unsuccessful BCG treatment and instead performance of radical cystectomy in the highest-risk patients, reducing unnecessary delay $[15,16]$.

In this study, we retrospectively evaluated the utility of preoperative NLR and other blood count-derived inflammatory markers in prediction of BCG failure in the population of high-risk NMIBC patients. Unlike in previous studies, retrospective definition of BCG failure as the endpoint was determined based on the updated EAU 2019 guidelines [1, 22, 23]. We hypothesized that a systemic inflammatory response might negatively im- 
A

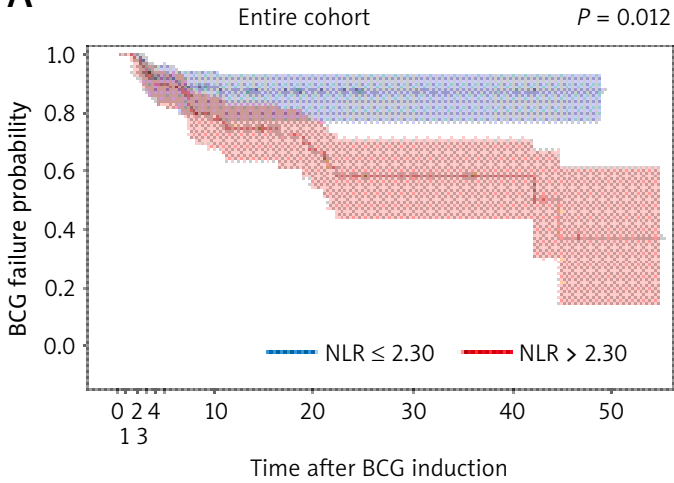

$\mathrm{C}$

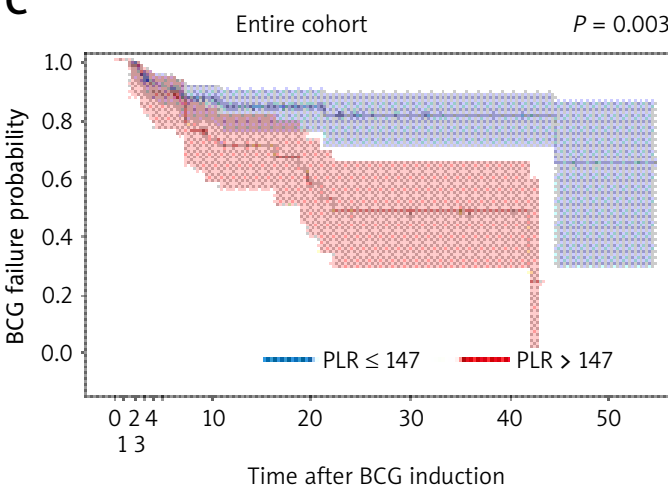

E

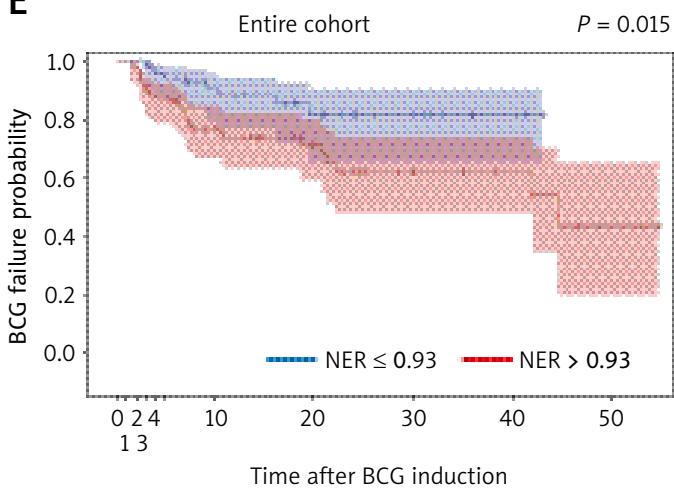

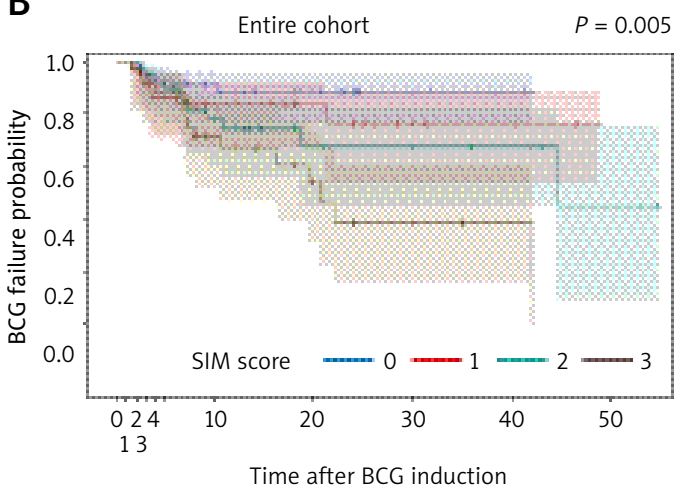

D



$\mathrm{F}$

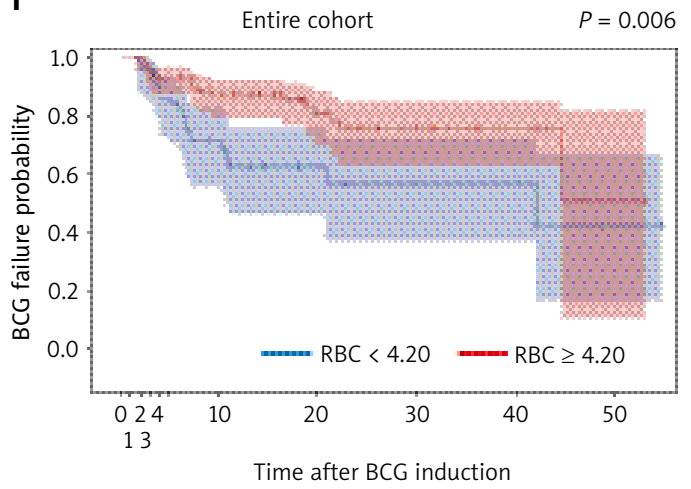

Figure $1 \mathrm{~A}$. Bacillus Calmette-Guérin (BCG) failure-free survival stratified by blood count-derived inflammatory markers (A - neutrophil-to-lymphocyte ratio - NLR, B - systemic inflammatory marker score - SIM score, C - plateletto-lymphocyte ratio - PLR, D - lymphocyte-to-monocyte ratio - LMR, E - neutrophil-to-erythrocyte - NER) and $\mathbf{F}$ - red blood cell count - entire cohort

pact the local response to BCG antigens, which is inevitable to obtain the desired cancer eradication.

Our study demonstrates that high preoperative NLR (> 2.3) and systemic inflammatory marker score are associated with shorter time to BCG failure. Importantly, we identified subgroups in which NLR implementation into risk-stratification tools might be especially relevant. Since high NLR values helped to further sub-stratify patients with the highest CUETO progression score, it might serve as an adjunct to currently used risk scores, especially when immediate radical cystectomy is being considered. Obviously in such scenarios, abnormal values of inflammatory markers must always be contextualized within the patient's clinical characteristics and histopathologic tumour features and cannot be used as single decisive factor.

Neutrophil-to-lymphocyte ratio marks time to BCG failure in papillary tumours ( $\mathrm{Ta}$ and $\mathrm{T} 1$ ) and tended to mark it in solitary CIS, which indicates its universal implementation regardless of staging. Furthermore, high preoperative NLR ( $>$ 2.3) appeared to be a negative prognostic 
Table II. Predictors of BCG failure. Univariate (A) and multivariate (B) analysis - logistic regression

A

\begin{tabular}{|c|c|c|}
\hline Variable & OR $(95 \% \mathrm{Cl})$ & $P$-value \\
\hline Male & $0.62(0.28-1.36)$ & NS \\
\hline Age & $1.02(0.98-1.06)$ & NS \\
\hline \multicolumn{3}{|l|}{ Tumour category } \\
\hline $\mathrm{Ta}$ & - & - \\
\hline $\mathrm{T} 1$ & $1.34(0.50-3.57)$ & NS \\
\hline $\mathrm{CIS}$ & $1.27(0.36-4.50)$ & NS \\
\hline Concomitant CIS & $1.87(0.82-4.25)$ & NS \\
\hline $\begin{array}{l}\text { Grade (high-grade } \\
\text { vs. low-grade) }\end{array}$ & $2.84(0.35-22.85)$ & NS \\
\hline Multiplicity & $2.65(1.28-5.49)$ & 0.009 \\
\hline $\begin{array}{l}\text { Tumour size }(\geq 3 \mathrm{~cm} \\
\text { vs. }<3 \mathrm{~cm})\end{array}$ & $1.29(0.60-2.79)$ & NS \\
\hline Recurrent tumour & $1.36(0.65-2.87)$ & NS \\
\hline \multicolumn{3}{|l|}{ re-TURBT status } \\
\hline No re-TURBT & - & - \\
\hline No tumour & $0.32(0.12-0.87)$ & 0.011 \\
\hline Residual tumour & $1.04(0.46-2.36)$ & 0.107 \\
\hline Diabetes mellitus & $1.21(0.53-2.74)$ & NS \\
\hline $\begin{array}{l}\text { CUETO recurrence risk } \\
\text { score }\end{array}$ & $1.18(1.03-1.35)$ & 0.016 \\
\hline $\begin{array}{l}\text { CUETO progression risk } \\
\text { score }\end{array}$ & $1.20(1.01-1.43)$ & 0.041 \\
\hline $\begin{array}{l}\text { Concomitant upper } \\
\text { tract urothelial cancer }\end{array}$ & $1.97(0.63-6.15)$ & 0.098 \\
\hline $\mathrm{RBC}$ & $0.56(0.31-1.02)$ & 0.056 \\
\hline NLR & $1.22(1.00-1.50)$ & 0.056 \\
\hline PLR & $1.01(1.001-1.015)$ & 0.02 \\
\hline NER & $1.87(0.94-3.72)$ & 0.074 \\
\hline$N L R>2.3$ & $3.33(1.48-7.52)$ & 0.004 \\
\hline PLR > 147 & $3.16(1.52-6.57)$ & 0.002 \\
\hline$L M R<2.55$ & $2.75(1.33-5.70)$ & 0.007 \\
\hline NER > 0.93 & $3.07(1.36-6.92)$ & 0.007 \\
\hline \multicolumn{3}{|c|}{ Systemic inflammatory markers score } \\
\hline 1 vs. 0 & $2.15(0.67-6.94)$ & 0.004 \\
\hline 2 vs. 0 & $3.61(1.22-10.69)$ & \\
\hline 3 vs. 0 & $6.41(2.22-18.55)$ & \\
\hline
\end{tabular}

NLR - neutrophil count/lymphocyte count, NER - neutrophil count/ red blood cell count, PLR - platelet count/lymphocyte count, $L M R$ - lymphocyte count/monocyte count, SIM score $=N L R+P L R$ $+L M R$
B

\begin{tabular}{|c|c|c|}
\hline Variable & OR $(95 \% \mathrm{Cl})$ & $P$-value \\
\hline \multicolumn{3}{|l|}{ NLR-based model } \\
\hline $\begin{array}{l}\text { CUETO recurrence } \\
\text { risk score }\end{array}$ & 1.19 (1.04-1.37) & 0.014 \\
\hline NLR & $1.24(1.01-1.53)$ & 0.038 \\
\hline \multicolumn{3}{|l|}{ PLR-based model } \\
\hline $\begin{array}{l}\text { CUETO recurrence } \\
\text { risk score }\end{array}$ & $1.18(1.03-1.35)$ & 0.014 \\
\hline PLR & 1.01 (1.001-1.014) & 0.023 \\
\hline \multicolumn{3}{|l|}{ NER-based model } \\
\hline $\begin{array}{l}\text { CUETO recurrence } \\
\text { risk score }\end{array}$ & $1.20(1.04-1.38)$ & 0.014 \\
\hline NER & $2.08(1.02-4.23)$ & 0.039 \\
\hline \multicolumn{3}{|l|}{ LMR-based model } \\
\hline $\begin{array}{l}\text { CUETO recurrence } \\
\text { risk score }\end{array}$ & $1.18(1.03-1.36)$ & 0.014 \\
\hline LMR & $0.83(0.61-1.12)$ & 0.22 \\
\hline \multicolumn{3}{|l|}{ SIM score-based model } \\
\hline $\begin{array}{l}\text { CUETO recurrence } \\
\text { risk score }\end{array}$ & 1.18 (1.02-1.37) & 0.020 \\
\hline \multicolumn{3}{|l|}{ SIM score } \\
\hline 1 & $2.07(0.63-6.79)$ & 0.003 \\
\hline 2 & $3.72(1.23-11.22)$ & \\
\hline 3 & $6.24(2.12-18.34)$ & \\
\hline 0 & 1 & \\
\hline
\end{tabular}

$N L R$ - neutrophil count/lymphocyte count, NER - neutrophil count/ red blood cell count, $P L R$ - platelet count/lymphocyte count, $L M R-$ lymphocyte count/monocyte count, SIM score $=N L R+P L R+L M R$

factor, especially in patients with recurrent tumour, single tumour, when tumour size $<3 \mathrm{~cm}$ and when $\mathrm{RBC} \geq 4.2 \times 10^{12} / \mathrm{l}$. Neutrophil-to-lymphocyte ratio remains a predictor of $B C G$ failure in the subgroup of patients without diabetes mellitus or metabolic syndrome, which indicates that common systemic diseases such as diabetes do not constitute a confounder in the analysis. Identification of specific subgroups in which NLR might be especially efficient requires further studies on a larger cohort of patients.

In the multivariate model, NLR remained a significant adjunct to the CUETO recurrence risk score when predicting BCG failure. Similarly to other studies we propose NLR incorporation into risk stratification tools for high-risk NMIBC to improve their accuracy [18, 24-26]. Importantly, several studies have investigated the prognostic role of high preoperative NLR in the cohort 
A

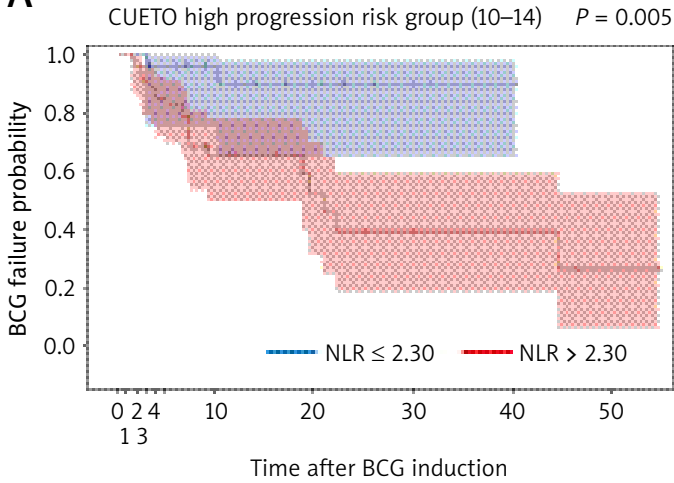

C

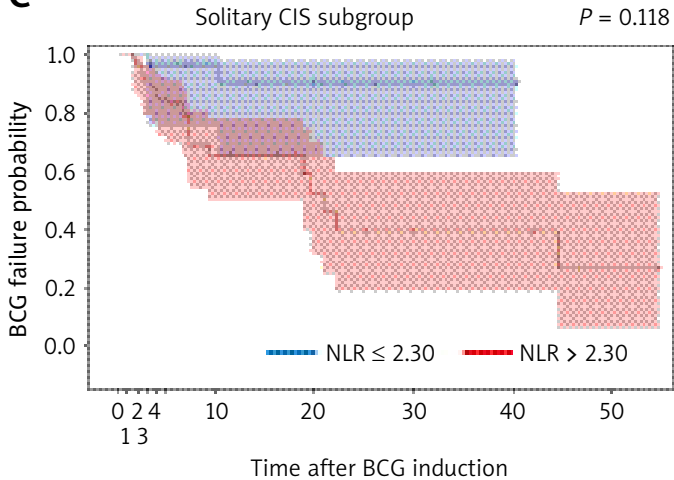

E

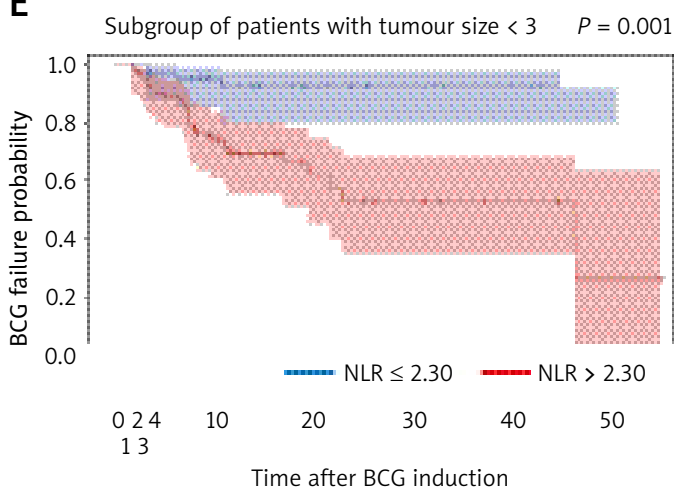

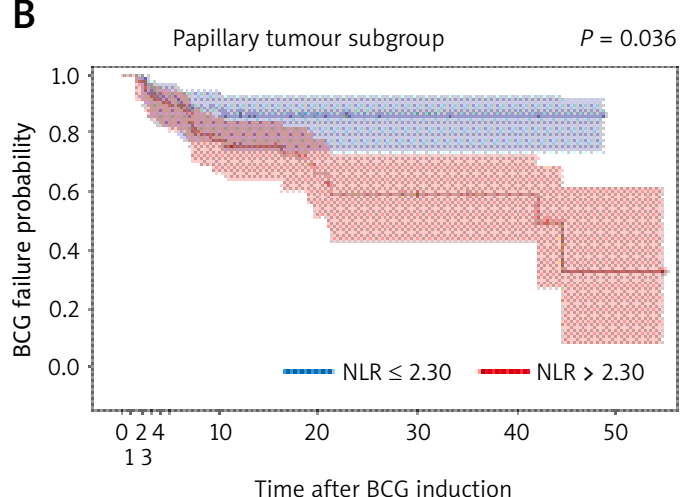

D

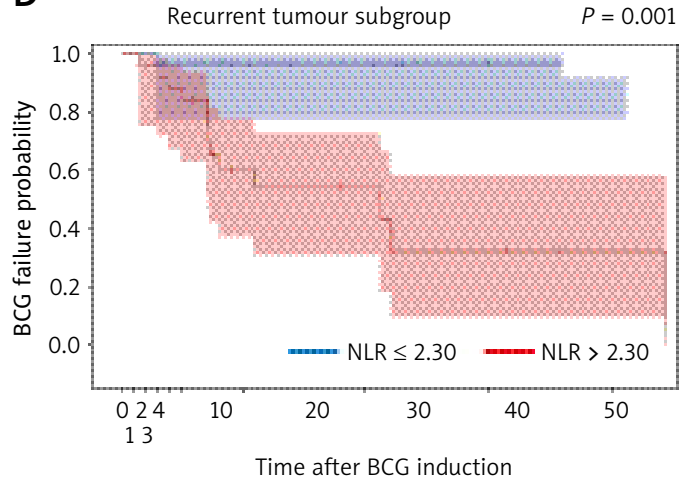

F

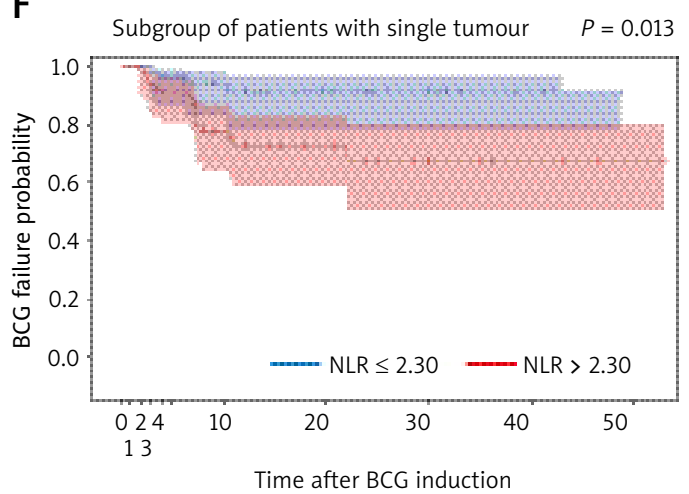

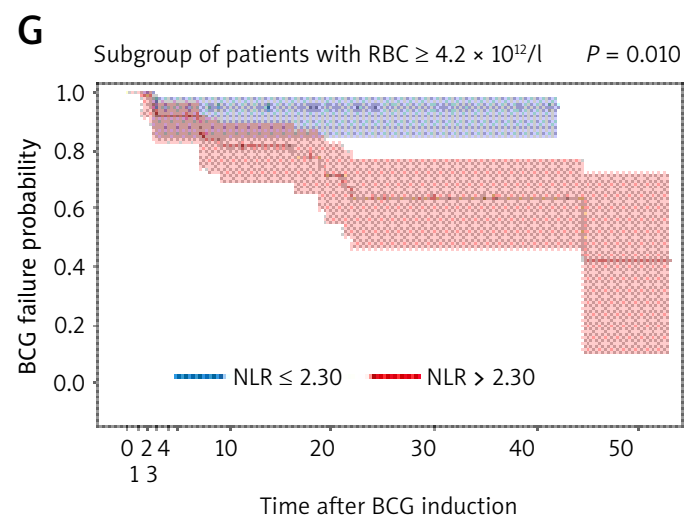

Figure 1 B. Bacillus Calmette-Guérin (BCG) failure-free survival stratified by NLR - subgroup analysis 
of BCG treated NMIBC, but they focused only on recurrence and progression risk [24, 25, 27, 28]. Only one study focused on its value in BCG failure prediction [22]. Notably, patients without tumour progression to MIBC are also classified as BCG failures when high-grade recurrence is diagnosed [1]. Therefore, prediction of disease progression might not always be the equivalent of BCG failure prediction, which covers wider spectra of clinical situations (high-grade recurrence). Of note, aforementioned studies reporting the value of NLR in recurrence prediction $[24,25,27,28]$ do not discriminate high and low-grade recurrence, which is of utmost significance to diagnose BCG immunotherapy failure and implement a new treatment strategy. Therefore NLR association with tumour recurrence and progression demonstrates only partial utility of this marker. In our study, we focused particularly on BCG failure defined as BCG unresponsive or progression to MIBC. Using such a definition we attempted to denote a group of patients for whom BCG does not provide any benefit. For such patients only radical cystectomy remains a true option $[1,6]$. Therefore, we believe that our detailed analysis offers a more precise view of NLR utility in the stratification of patients who are at high risk of not benefiting from BCG immunotherapy.

Association of residual tumour presence in reTURBT and BCG failure was not significant $(p=0.11)$ and was not included in the final multivariate model. However, such a potential association underlies the pivotal role of re-TURBT in NMIBC management [17, 21, 29]. It has been recently reported that re-TURBT pathology constitutes an independent prognostic factor for NMIBC recurrence and progression [30]. Further evaluation of the impact of re-TURBT pathology on prognosis in high-risk BCG-treated patients is warranted.

We have also attempted to determine an association between other blood cell count-derived parameters and BCG failure. High PLR ( $>147)$ and low LMR $(<2.55)$ were associated with shorter time to BCG failure. Recently, it has been shown that PLR and LMR provide additional information to NLR when combined together in the SIM score [19]. Association of high SIM score with recurrence and progression in T1HG NMIBC was reported [19]. In our analysis, SIM score remained a significant predictor of BCG failure in the uni- and multivariable model. Consequently, SIM score constitutes a promising parameter, which mirrors the level of systemic inflammation and has predictive value for time to BCG failure.

We found that patients with a low RBC count $\left(<4.2 \times 10^{12} / l\right)$ had a shorter time to BCG failure. It can be suspected that the predictive value of erythropoietic parameters is related to their ability to mirror the state of chronic inflammation, which is known to impair erythropoiesis and shorten the erythrocyte half-life [31]. This corresponds with the hypothesis that systemic inflammation is associated with a poorer BCG response. To the best of our knowledge, this is the first report on the association of red blood cell count and neutrophil-to-erythrocyte ratio with BCG failure. Incorporation of erythrocyte and neutrophil counts into one derived index might combine the advantages of both parameters in terms of their predictive values. On the other hand, the impact of preoperative (pre-TURBT) anaemia on cancer-specific and overall survival in NMIBC patients has been previously reported [20, 32, 33].

Study limitations including selection bias result from its retrospective design. Due to methodological issues, the follow-up time was limited to 6 months from the last exposure to BCG. Therapy interruption due to multiple factors (age, comorbidities, intolerance, non-compliance) led to suboptimal maintenance in a significant number of cases. The probability of missing a small tumour during the initial TURBT and its further identification as recurrence during BCG is limited due to re-TURBT performance (71.5\% patients) and strict cystoscopic follow-up.

In conclusion, prediction of BCG immunotherapy outcomes remains a challenge, which should be overcome in order to achieve durable cancer control in patients with high-risk NMIBC. We found that simple blood count-derived markers NLR, PLR, NER, LMR and SIM score - might be utilized to predict $B C G$ immunotherapy failure. Since NLR, PLR, NER and SIM score predict BCG failure independently from the CUETO scoring system, they can be easily implemented in everyday practice and aid clinical decision-making.

\section{Conflict of interest}

The authors declare no conflict of interest.

\section{References}

1. Babjuk M, Burger M, Comperat EM, et al. European Association of Urology guidelines on non-muscle-invasive bladder cancer (TaT1 and carcinoma in situ) - 2019 update. Eur Urol 2019; 76: 639-57.

2. Sylvester RJ, van der Meijden AP, Oosterlinck W, et al. Predicting recurrence and progression in individual patients with stage Ta $T 1$ bladder cancer using EORTC risk tables: a combined analysis of 2596 patients from seven EORTC trials. Eur Urol 2006; 49: 466-5; discussion 75-7.

3. Xylinas E, Kent M, Kluth L, et al. Accuracy of the EORTC risk tables and of the CUETO scoring model to predict outcomes in non-muscle-invasive urothelial carcinoma of the bladder. Br J Cancer 2013; 109: 1460-6.

4. Sylvester RJ, Brausi MA, Kirkels WJ, et al. Long-term efficacy results of EORTC genito-urinary group randomized 
phase 3 study 30911 comparing intravesical instillations of epirubicin, bacillus Calmette-Guerin, and bacillus Calmette-Guerin plus isoniazid in patients with intermediate- and high-risk stage Ta T1 urothelial carcinoma of the bladder. Eur Urol 2010; 57: 766-73.

5. Poletajew S, Krajewski W, Gajewska D, et al. Prediction of the risk of surgical complications in patients undergoing monopolar transurethral resection of bladder tumour - a prospective multicentre observational study. Arch Med Sci 2020; 16: 863-70.

6. Kamat AM, Sylvester RJ, Bohle A, et al. Definitions, end points, and clinical trial designs for non-muscle-invasive bladder cancer: recommendations from the International Bladder Cancer Group. J Clin Oncol 2016; 34: 1935-44

7. Sylvester RJ. Bacillus Calmette-Guerin treatment of nonmuscle invasive bladder cancer. Int J Urol 2011; 18: 113-20.

8. Poletajew S, Zapala P, Radziszewski P. Safety and efficacy of intravesical Bacillus Calmette-Guerin immunotherapy in patients with non-muscle-invasive bladder cancer presenting with asymptomatic bacteriuria: a systematic review. Urol Int 2017; 99: 1-5.

9. van der Meijden AP, Sylvester RJ, Oosterlinck W, Hoeltl W, Bono AV, Group EG-UTC. Maintenance Bacillus CalmetteGuerin for Ta T1 bladder tumors is not associated with increased toxicity: results from a European Organisation for Research and Treatment of Cancer GenitoUrinary Group Phase III Trial. Eur Urol 2003; 44: 429-34.

10. Brausi $M$, Oddens J, Sylvester $R$, et al. Side effects of Bacillus Calmette-Guerin (BCG) in the treatment of intermediate- and high-risk Ta, $\mathrm{T} 1$ papillary carcinoma of the bladder: results of the EORTC genito-urinary cancers group randomised phase 3 study comparing one-third dose with full dose and 1 year with 3 years of maintenance BCG. Eur Urol 2014; 65: 69-76.

11. Gheorghe GCG, Gheorghe F, Bratu OG, Bacalbasa N, Bungau S, Diaconu C. Fever of unknown origin. Rom J Mil Med 2020; 123: 213-8.

12. Scarneciu I, Bungau S, Lupu AM, et al. Efficacy of instillation treatment with hyaluronic acid in relieving symptoms in patients with BPS/IC and uncomplicated recurrent urinary tract infections - long-term results of a multicenter study. Eur J Pharm Sci 2019; 139: 105067.

13. Zuiverloon TC, Zwarthoff EC. Predicting response to in travesical Bacillus Calmette-Guerin immunotherapy: are we moving forward? Eur Urol 2016; 69: 201-2.

14. Slusarczyk A, Zapala P, Zapala L, Piecha T, Radziszewski P. Prediction of BCG responses in non-muscle-invasive bladder cancer in the era of novel immunotherapeutics. Int Urol Nephrol 2019; 51: 1089-99.

15. Schrier BP, Hollander MP, van Rhijn BW, Kiemeney LA, Witjes JA. Prognosis of muscle-invasive bladder cancer: difference between primary and progressive tumours and implications for therapy. Eur Urol 2004; 45: 292-6.

16. Moschini $M$, Sharma V, Dell'oglio $P$, et al. Comparing long-term outcomes of primary and progressive carcinoma invading bladder muscle after radical cystectomy. BJU Int 2016; 117: 604-10.

17. Fernandez-Gomez J, Madero R, Solsona E, et al. Predicting nonmuscle invasive bladder cancer recurrence and progression in patients treated with bacillus Calmette-Guerin: the CUETO scoring model. J Urol 2009; 182: 2195-203.

18. Vartolomei MD, Porav-Hodade D, Ferro M, et al. Prognostic role of pretreatment neutrophil-to-lymphocyte ratio (NLR) in patients with non-muscle-invasive bladder cancer (NMIBC): a systematic review and meta-analysis. Urol Oncol 2018; 36: 389-99.
19. Cantiello F, Russo GI, Vartolomei MD, et al. Systemic inflammatory markers and oncologic outcomes in patients with high-risk non-muscle-invasive urothelial bladder cancer. Eur Urol Oncol 2018; 1: 403-10.

20. Soria F, Moschini M, Abufaraj M, et al. Preoperative anemia is associated with disease recurrence and progression in patients with non-muscle-invasive bladder cancer. Urol Oncol 2017; 35: 113.e9-e14.

21. Cambier S, Sylvester RJ, Collette L, et al. EORTC nomograms and risk groups for predicting recurrence, progression, and disease-specific and overall survival in non-muscle-invasive stage ta-t1 urothelial bladder cancer patients treated with 1-3 years of maintenance bacillus Calmette-Guerin. Eur Urol 2016; 69: 60-9.

22. Racioppi M, Di Gianfrancesco L, Ragonese M, Palermo G, Sacco E, Bassi PF. Can Neutrophil-to-Lymphocyte ratio predict the response to BCG in high-risk non muscle invasive bladder cancer? Int Braz J Urol 2019; 45: 315-24.

23. Gual Frau J, Palou J, Rodriguez O, Parada R, Breda A, Villavicencio $H$. Failure of bacillus Calmette-Guerin therapy in non-muscle-invasive bladder cancer: definition and treatment options. Arch Esp Urol 2016; 69: 423-33.

24. Vartolomei MD, Ferro M, Cantiello F, et al. Validation of neutrophil-to-lymphocyte ratio in a multi-institutional cohort of patients with T1G3 non-muscle-invasive bladder cancer. Clin Genitourin Cancer 2018; 16: 445-52.

25. D’Andrea D, Moschini M, Gust K, et al. Prognostic role of neutrophil-to-lymphocyte ratio in primary non-muscleinvasive bladder cancer. Clin Genitourin Cancer 2017; 15 : e755-e64.

26. Getzler I, Bahouth Z, Nativ O, Rubinstein J, Halachmi S. Preoperative neutrophil to lymphocyte ratio improves recurrence prediction of non-muscle invasive bladder cancer. BMC Urol 2018; 18: 90.

27. Mano R, Baniel J, Shoshany O, et al. Neutrophil-to-lymphocyte ratio predicts progression and recurrence of non-muscle-invasive bladder cancer. Urol Oncol 2015; 33: 67.e1-7.

28. Mbeutcha A, Shariat SF, Rieken M, et al. Prognostic significance of markers of systemic inflammatory response in patients with non-muscle-invasive bladder cancer. Urol Oncol 2016; 34: 483.e17-e24.

29. Zapala P, Dybowski B, Poletajew S, Bialek L, Niewczas A, Radziszewski P. Clinical rationale and safety of restaging transurethral resection in indication-stratified patients with high-risk non-muscle-invasive bladder cancer. World J Surg Oncol 2018; 16: 6.

30. Zhang G, Steinbach D, Grimm MO, Horstmann M. Utility of the EORTC risk tables and CUETO scoring model for predicting recurrence and progression in non-muscle-invasive bladder cancer patients treated with routine second transurethral resection. World J Urol 2019; 37: 2699-705.

31. Weiss G, Ganz T, Goodnough LT. Anemia of inflammation. Blood 2019; 133: 40-50.

32. Kang M, Jeong CW, Kwak C, Kim HH, Ku JH. Preoperative neutrophil-lymphocyte ratio can significantly predict mortality outcomes in patients with non-muscle invasive bladder cancer undergoing transurethral resection of bladder tumor. Oncotarget 2017; 8: 12891-901.

33. Tang G, Zhen Y, Xie W, et al. Preoperative hemoglobin-platelet ratio can significantly predict progression and mortality outcomes in patients with T1G3 bladder cancer undergoing transurethral resection of bladder tumor. Oncotarget 2018; 9: 18627-36. 